\title{
Features of propagation by seeds of rare and endangered plant species in culture
}

\author{
Tatyana Elisafenko ${ }^{1 *}$, and Victoria Yakk $^{1}$ \\ ${ }^{1}$ Central Siberian Botanical Garden of the SB RAS, Zolotodolinskaya Str., 101, Novosibirsk, 630090, \\ Russia
}

\begin{abstract}
The article is devoted to the conservation of rare and endangered species in a culture The problems and features of reproduction by seeds of these species were been considered. The stages were been distinguished - preparatory (informational), seed collection, drying, peeling, storage, germination, genetic identification. Approaches to seed collection and storage were been presented. It is proposed to use a refrigeration chamber $\left(+4^{\circ} \mathrm{C}\right)$ for long-term storage of seeds.
\end{abstract}

The conservation of plant biodiversity is part of the global problem of the biosphere function. At present, the formation of stable introduction populations in collections of rare and endangered species (RE) in introduction centers is becoming relevant. Such collections include the plants in the open ground, the tissue culture and seed collection. It is the foundation for exchange between centers and for the restoration of natural populations [1].

The specificity of the introduction of RE species is the removal of a limited amount of material from nature or obtaining from introduction centers. Seeds, live plants or their vegetative parts are being used for introduction in the present. Unfortunately, biotechnological methods are not sufficiently applied. Using these methods, a large number of regenerants can be obtained from a small number of source material $[2,3]$. One of the results of creating a collection of RE species is the formation of stable introduction populations with a large number of individuals. It prevents the constant removal of material from natural conditions. Propagation of plants is one of the main directions at the stage of primary introduction. It should be carried out at the same time with a complex of studies: detection of the characteristics of seed reproduction, ontogenes description, phenological observations, molecular genetic analysis. As a result of this approach, it is possible to identify the causes of rarity, to determine the practicability of introduction, and to develop recommendations for growing RE species. In each type of collection (open ground, seed collection, tissue culture), certain conditions must be selected to preserve the material. Knowledge of the features of fruiting and the latent period of the RE species (germination conditions, biological and introduction-profitability longevity, reaction to various storage conditions) determine the successful use of the seed collection. Methods and problems of propagation of RE species by seeds in the culture are considered in this article.

Reproduction by seeds includes several stages: a preparatory stage (information about the species biological characteristics), collecting seeds, drying, purification, storage,

* Corresponding author: tveli@ngsl.ru 
germination, genetic identification. During the preparatory stage, the characteristic of fruiting and the presence of seed dormancy are been noted. When collecting seeds, various techniques are used depending on the biological characteristics, for example, isolation of unripe fruits. As the seeds of milk ripeness of plants of the genus Fornicium (Stemmacanta, Asteraceae) are intensively eaten by birds, so it is necessary at this time the baskets are isolated the until the seeds are fully ripe. The baskets are sufficiently been covered by bags of light tissue [4]. Many species of the genus Viola are mechanochores, and when their fruits dry out, it crack and seeds are scattered up to 3-5 m. Therefore, seeds of plants of this genus must be collected in the morning before the mature fruits dry out. Their maturity is determined by the vertical position of fruit stalk. [5]. Often from plants are harvested fruits, not seeds. It is necessary to determine the percentage of fruit production, potential and real seed productivity per individual, shoot or fruit during the collection and during seed cleaning [6]. Therefore, the fruits and seeds are gathered out according to the tasks in different packages. Crop labeling during collection, drying and storage is an important component for determining seed productivity and documentation the seed collection. This will allow to obtain the necessary information about the biology of the species at the first stages of introduction and to monitor the availability of material for experiments and reproduction. The havest of some species is advisable to store in the form of fruits or parts of them (Lamiaceae). Fleshy fruits harden upon drying; for such plants, seeds must be extracted for further storage. Most often, seeds of RE species are presented in small quantities, so cleaning is carried out manually.

Long-term storage of seeds is one of the most common and effective approaches to the conservation of most plant species. The easiest way to store seeds is at room conditions in paper bags away from heating devices. It is not optimal storage conditions for many plant species. The germination of their seeds decreases sharply with keeping period. One of the reasons for the loss of seed viability is the accumulation of gene mutations that occur due to chromosome breaks or oxidation of storage compound [7]. A large number of works are devoted to the development of storage methods and the study of seed viability during longterm storage $[4,8,9,10,11]$. The information presented in the literature relates mainly to ornamental, forage, food and medicinal plants $[12,13,14]$. Data on germination, about storage conditions and longevity of seeds of RE species are few, but for most of these species are absent [15]. It is necessary to study the quality of seeds under various storage conditions. A promising technology is cryo-conservation - deep freezing of seeds in liquid nitrogen $\left(-196^{\circ} \mathrm{C}\right)$. For this purpose, specialized storage facilities are created all over the world, mostly for cultivated plants [7]. However, some species cannot be stored in this way. For example, seeds containing a significant amount of storage compound and having large cotyledons (for example, the genus Fornicium) do not tolerate cryo-conservation [11]. In addition, most introduction centers do not have the necessary equipment. The International Council of Botanic Gardens for Plant Conservation proposed two temperature regimes for storing seeds: low positive temperatures $\left(+5^{\circ} \mathrm{C}\right)$ and shallow freezing $\left(-20^{\circ} \mathrm{C}\right)$. These temperature conditions slow down the metabolic processes and extend the viability of the seeds. According to the results of our research on the influence of the seed storage conditions for a number of RE species, we proposed to store seeds at $+4{ }^{\circ} \mathrm{C}$ (refrigerator) [16]. Seeds of some species after storage for several years at low temperatures subsequently germinate without cold stratification. It must be borne in mind that the seeds of some species have no seed dormancy, and germination is lost after a few days. This is characteristic of woody species, for example, Chosenia arbutifolia (Pall.) A.Skvorts [4]. The seeds of such plants should be sown in the soil after their harvesting, or laid for germination in accordance with the rules presented below.

Determining seed longevity is an important study of RE species. Biological (germination rate of more than $0 \%$ ) and economic (preservation of conditional germination) 
seed longevity is distinguished in seedage. We suggested that the period of preservation of laboratory germination of more than $10 \%$ should be defined as introduction-profitability longevity of seeds [17]. Such germination allows using the laboratory-greenhouse-soil method to support most species in the collection.

The paucity of the source material and the small number of seeds complicates the reproduction of RE species. Therefore, the determination of the conditions for seed germination and the development of methodological techniques are especially important. There is high mortality in the pregenerative period for many plant species, especially at the seedling stage. We use the laboratory-greenhouse-soil method for propagation with a small number of seeds, which allows us to preserve individuals [18]. Techniques used for the propagation of RE species by this method are presented in a number of works [4, 5]. Not all RE species can be propagated in this way. We found that Caragana jubata (Pall.) Poiret (Fabaceae), Atraphaxis frutescens (L.) C. Koch (Polygonaceae), Stelleropsis altaica (Thieb.) Pobed. (Thymelaeceae) it is advisable to propagate by sowing seeds into the ground in autumn. Seeds of some species germinate after a long period with variable temperatures and with long cold stratification (more than 1 year), for example, Cotoneaster lucidus Schlecht. (Rosaceae). Plants of some medium-resistant species in the culture conditions do not always form mature seeds during one growing season. In this case, to maintain such species in the collection, it is necessary to grow seedlings in phytochambers or greenhouses during the winter. The individuals are planted out in the virginal stage and this makes it possible to obtain seeds in the introduction conditions.

In the cultural conditions, long life cycle of many species is reduced and self-sown plant is absent. Therefore, such populations must be regularly replenished. For example, Enneapogon borealis (Griseb.) Honda (Poaceae) under natural conditions is a biennial, but in culture it becomes an annual, while it does not form self-sown. Sowing the seeds of this species in the ground in the spring (May-June), it let to obtain mature seeds in September. This species is represented in the collection "Rare and Endangered Species of Siberian Plants" of the Central Siberian Botanical Garden (Novosibirsk) since 1982.

The use of molecular genetic methods allows researcher to identify possible genetic processes occurring in populations during cultivation of RE species for a long time, as well as during storage of their seeds. These methods are also necessary for the certification and identification of the source material.

The limited territory of the collection site does not allow researcher to present the species with samples from various points of the area. Therefore, if studies of interpopulation variability of a particular species are not supposed, it is advisable to create hybrid populations. Growing individuals of the same species from different coenopopulations allows expanding the diversity of genotypes, which will contribute to the heterogeneity of the introduction populations and its stability in culture. It is important to identify the source of genetic material and such hybrid populations.

Thus, in the culture conditions, the propagation by seeds of rare and endangered species includes the following stages: preparatory (informational), seed harvesting, drying, purification, storage, germination, genetic identification. The preparatory phase is of particular importance. It allows you to rationally plan further work and prevent the loss of seed. For long-term storage, seeds should be stored in a refrigerator $\left(+4{ }^{\circ} \mathrm{C}\right)$.

The study is carried out according to of the state assignment of the Central Siberian Botanical Garden of the Siberian Branch of the Russian Academy of Sciences state registration no. AAAA-A17117012610051-5 under the project "Assessment of the morphogenetic potential of plant populations of North Asia by experimental methods» and applying materials bioresource scientific scientific collection of the Central Scientific and Biological Center SB RAS, "Collection of living plants in open and closed ground" USU 440534. 


\section{References}

1. T.V. Elisafenko, O.V. Dorogina, T.I. Novokova, Botany in the modern world, (Makhachkala, 2018)

2. A.A. Erst, N.S Zvyagina, T.I. Novokova, O.V. Dorogina, Russ. J. Genet., 51, 188-193 (2015)

3. D.S. Muraseva, N.S Zvyagina, T.I. Novokova, O.V. Dorogina, Vavilov journal of genetics and breeding, 21, 554-560 (2017)

4. G.P. Semenova, Rare and endangered flora species of Siberia: biology and protection (Novosibirsk, 2007)

5. T.V. Elisafenko, Rastitel'nyj mir Aziatskoj Rossii, 2, 66-72 (2012)

6. I.V. Vaynagiy, Bot. Zhurn., 59, 826-831 (1974)

7. K. Sax, H.J. Sax, Radiat. Bot., 4, 37-41 (1964)

8. C. Nichols, Spontaneous chromosome aberration in allium.Genetics, 26, 89-100 (1941)

9. V.P. Viktorov, Teacher of the XXI century, 211 (2009)

10. V.L. Tikhonova, I.A. Smirnov, E.V. Morozova, Newsletter of the Council of Botanical Gardens of Russia and the dedication of the International Council of Botanical Gardens on Plant ProtectiIon, 12, 90-94 (2001)

11. M.M. Ishmuratova, K.G. Tkachenko, Seeds of herbaceous plants: features of the latent period, use in introduction and reproduction in vitro (Ufa, 2009)

12. N.A. Emelyanova, Seed viability (Moscow, 1978)

13. R.I. Zlotin, E.V. Yasny, Proceedings of the Academy of Sciences of the USSR. Geographical series, 2, 76-88 (1992)

14. GOST 12038-84 Seeds of agricultural crops. Germination determination methods (Moscow, 2011)

15. M.G. Nikolaeva, I.V. Lyazgunova, L.M Pozdova, Seed biology (St. Petersburg, 1999)

16. V.N Balakina, T.V. Elisafenko, Problems of Botany in Southern Siberia and Mongolia. (Barnaul, 2017)

17. T.V. Elisafenko, O.V. Dorogina, Cryopreservation of seeds: results and prospects (Novosibirsk, 2014)

18. G.P. Dyuryagina, Bot. Zhurn., 67, 679-687 (1982) 